\title{
Short Synthesis of New 13,16-Diazaestrone and 13,16-Diazaequilenin Analogs
}

\author{
J. A. Parihar and M. M. V. Ramana* \\ Department of Chemistry, University of Mumbai, Vidyanagari, Santacruz (E), Mumbai-400098, India
}

\begin{abstract}
Diferentes 3-[2-(3,4-diidro-1-naftil)etil]imidazolidina-2,4-dionas e 3-[2-(1-naftil)etil]imidazolidina-2,4-dionas, quando aquecidas em ácido polifosfórico a $150{ }^{\circ} \mathrm{C}$, sofrem ciclização quimioseletiva intramolecular resultado nos respectivos esteróides 13,16-diazaestrona e 13,16diazaequilenina.
\end{abstract}

Different 3-[2-(3,4-dihydro-1-naphthyl)ethyl]imidazolidine-2,4-diones and 3-[2-(1naphthyl)ethyl]imidazolidine-2,4-diones, when heated in polyphosphoric acid at $150{ }^{\circ} \mathrm{C}$, underwent chemoselective intramolecular cyclization to afford the 13,16-diazaestrone steroids and 13,16diazaequilenin steroids respectively.

Keywords: polyphosphoric acid, chemoselective cyclization, 13,16-diazaestrone steroids, 13,16diazaequilenin steroids

\section{Introduction}

Azasteroids are known to exhibit various biological properties which include analgesic, ${ }^{1}$ antiandrogenic, ${ }^{2}$ antiphlogistic, ${ }^{3}$ antimicrobial, ${ }^{4}$ antileukemic,${ }^{5}$ antifungal,${ }^{6}$ bactericide, ${ }^{7}$ antiestrogenic, ${ }^{8}$ antifertility, ${ }^{9}$ and cardiotonic and hypotensive activities. ${ }^{10}$ Moreover, some azasteroids act as neuromuscular blockers ${ }^{11}$ and inhibitors of 5- $\alpha$ reductase and androgen receptor binding. ${ }^{12}$ Recently we have reported ${ }^{13,14}$ the syntheses of 13,16-diazaestrone and 13,16-diazaequilenin analogs from the intramolecular cyclization of the corresponding 5-hydroxyimidazolidin2-ones. We now wish to report a new synthesis of the title compounds from intramolecular cyclization of the corresponding imidazolidine-2,4-diones.

\section{Results and Discussion}

Towards this end, the intramolecular cyclization of 3-[2-(3,4-dihydro-1-naphthyl)ethyl]imidazolidine-2,4dione ${ }^{13} \mathbf{1 a}$ was undertaken. All our attempts to cyclize 1a employing $\mathrm{POCl}_{3}$ and $\mathrm{P}_{2} \mathrm{O}_{5}$ in different solvents such as benzene, dichloroethane, toluene and xylene were unsatisfactory and gave the starting material back. Heating 1a with $\mathrm{POCl}_{3}$ in refluxing tetralin gave a brown solid, which was insoluble in all organic solvents and

* e-mail: mmvramana@indiatimes.com hence could not be characterized. Attempts to cyclize 1a in polyphosphoric acid (PPA) at $100{ }^{\circ} \mathrm{C}$ gave starting material back. When the temperature increased to $120^{\circ} \mathrm{C}$ the $\mathrm{TLC}\left(\mathrm{CHCl}_{3}-\mathrm{MeOH}, 96: 4\right)$ showed the formation of a new spot but the bulk of starting material remained intact even after $12 \mathrm{~h}$. The reaction was then carried out at $150{ }^{\circ} \mathrm{C}$, which led to consumption of the starting material in $6 \mathrm{~h}$ to give a yellow solid in $44 \%$ yield after aqueous work-up (Scheme 1). IR spectrum of the yellow solid showed the $\mathrm{N}-\mathrm{H}$ band at $3250 \mathrm{~cm}^{-1}$ and the ${ }^{1} \mathrm{H}$ NMR spectrum displayed a $1 \mathrm{H}$ broad singlet due to $\mathrm{N}-\mathrm{H}$ at $\delta 8.91$ and ${ }^{1} \mathrm{H}$ singlet due to vinylic proton at $\delta 6.70$ thus confirming the chemoselective formation of 13,16 diazaestrone 2a and not the corresponding 13,15diazasteroids. This may be explained on the basis of more electrophilic nature of carbonyl carbon of amide than urea. ${ }^{15,16}$ Proposed mechanism for intramolecular cyclization of 1a to give 2a is shown in Scheme 2. Following the above standardized protocol, intramolecular cyclization of other imidazolidine-2,4diones ${ }^{13,14} \mathbf{1 b}$-h were carried out to afford the corresponding 13,16-diazasteroids $\mathbf{2 b - h}$ in $42-59 \%$ yields (Scheme 1). It is important to notice that the earlier attempts by Schleigh et al. ${ }^{17}$ to synthesize 13 -azaestrone and 13-azaequilenin analogs by such one-step intramolecular cyclization of the corresponding pyrrolidine-2,5-diones under various acidic conditions were unsuccessful and gave either starting material back 


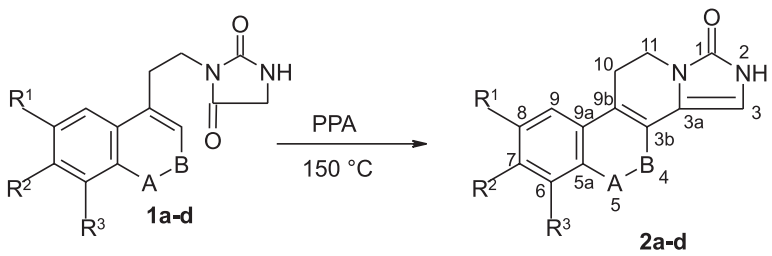

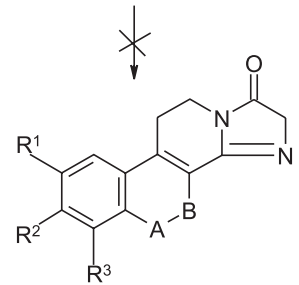

2a: $R^{1}, R^{2}, R^{3}=H ; A-B=C_{2}-C H_{2}$ 2b: $R^{1}=\mathrm{CH}_{3} ; \mathrm{R}^{2}, \mathrm{R}^{3}=\mathrm{H} ; \mathrm{A}-\mathrm{B}=\mathrm{CH}_{2}-\mathrm{CH}_{2}$ 2c: $R^{1}, \mathrm{R}^{2}, \mathrm{H} ; \mathrm{R}^{3}=\mathrm{Cl} ; \mathrm{A}-\mathrm{B}=\mathrm{CH}_{2}-\mathrm{CH}_{2}$ 2d: $R^{1}, R^{2}=\mathrm{CH}_{3} ; \mathrm{R}^{3}=\mathrm{H} ; \mathrm{A}-\mathrm{B}=\mathrm{CH}_{2}-\mathrm{CH}_{2}$ 2e: $R^{1}, R^{2}, R^{3}=H ; A-B=C H=C H$ 2f: $\mathrm{R}^{1}=\mathrm{CH}_{3} ; \mathrm{R}^{2}, \mathrm{R}^{3}=\mathrm{H} ; \mathrm{A}-\mathrm{B}=\mathrm{CH}=\mathrm{CH}$ 2g: $\mathrm{R}^{1}, \mathrm{R}^{2}, \mathrm{H} ; \mathrm{R}^{3}=\mathrm{Cl} ; \mathrm{A}-\mathrm{B}=\mathrm{CH}=\mathrm{CH}$ 2h: $R^{1}, \mathrm{R}^{2}=\mathrm{CH}_{3} ; \mathrm{R}^{3}=\mathrm{H} ; \mathrm{A}-\mathrm{B}=\mathrm{CH}=\mathrm{CH}$

Scheme 1. Syntheses of 13,16-diazaestrone and 13,16diazaequilenin analogs.

or a trace amount of uncharacterized solid material melting over a wide range.

In conclusion, the present work describes a new synthesis of 13,16-diazasteroids $\mathbf{2 a - h}$ by chemoselective cyclization of 1a-h in PPA. The method is short, general and utilizes easily accessible materials for the synthesis of the new 13,16-diazasteroids.

\section{Experimental}

Reagents were of LR grade and were used without further purification. Column chromatography was carried out using silica gel (S. D. Fine Chemicals, India) 60-120 mesh. Boiling point of Petroleum ether used was in the range of $60-80{ }^{\circ} \mathrm{C}$. The melting points (uncorrected) were determined on a Gallenkamp melting apparatus. The IR spectra (wavenumbers in $\mathrm{cm}^{-1}$ ) were recorded on a Shimadzu FTIR-4200 spectrometer either as oil film or $\mathrm{KBr}$ discs. UV spectra were recorded on a Shimadzu UV-Visible spectrophotometer UV-2100. ${ }^{1} \mathrm{H}$ NMR spectra were recorded on Varian EM-360L (60
MHz), Varian 200 (200 MHz), Bruker 300 (300 MHz) and Varian VR $(500 \mathrm{MHz})$ instruments in $\mathrm{CDCl}_{3}$ with tetramethylsilane as internal standard. Chemical shifts are given in ppm and coupling constants $(J)$ in $\mathrm{Hz}$. Elemental analyses were carried out on a Carlo Erba EA-1108 elemental analyser. The electron impact spectrum was recorded on a Katros MS-80.

General procedure for the synthesis of 13,16-diazasteroids $2 a-h$

A mixture of compound 1a-h (50 mg) and PPA (2 g) was heated at $150{ }^{\circ} \mathrm{C}$ for $6 \mathrm{~h}$. The reaction mixture was poured onto ice. It was extracted with EtOAc $(3 \times 25 \mathrm{~mL})$. The combined EtOAc extracts were washed with $10 \%$ $\mathrm{Na}_{2} \mathrm{CO}_{3}(2 \times 25 \mathrm{~mL})$, water $(2 \times 25 \mathrm{~mL})$ and then dried (anhydrous $\mathrm{Na}_{2} \mathrm{SO}_{4}$ ). Evaporation of solvent gave a brown residue, which was purified by column chromatography (basic alumina, $\mathrm{CHCl}_{3}-\mathrm{MeOH}, 95: 5$ ) to afford the corresponding compound $\mathbf{2 a - h}$.

4, 5, 10, 11 -Tetrahydro-2H-benzo[f]imidazo[5,1-a]isoquinolin-1-one (2a). Yield 44\%, mp 242-245 ${ }^{\circ} \mathrm{C}$ (decomp.). IR (KBr) $v_{\max } / \mathrm{cm}^{-1}: 1680(\mathrm{C}=\mathrm{C}), 1715(\mathrm{C}=\mathrm{O})$, 3250 (N-H). ${ }^{1} \mathrm{H} \mathrm{NMR}\left(\mathrm{CDCl}_{3}, 300 \mathrm{MHz}\right)$ : d 2.43 (t, 2H, J 6.5, H-4), 2.86 (t, 2H, J 6.5, H-5), 3.44 (t, 2H, J 7.8, H-10), 4.01 (t, $2 \mathrm{H}, J 7.8, \mathrm{H}-11), 6.70$ (s, 1H, C=C-H), 7.45-7.80 (m, 4H, Ar-H), 8.91 (s, 1H, N-H). UV $\left(\mathrm{CHCl}_{3}\right) \lambda_{\text {max }} / \mathrm{nm}(\log \varepsilon): 288$ (3.97), 307 (3.89), 322 (3.81). Analysis calc. for $\mathrm{C}_{15} \mathrm{H}_{14} \mathrm{~N}_{2} \mathrm{O}: \mathrm{C}, 75.61$; H, 5.92; N, 11.76; Found: C, 75.53; H, 5.97; N, 11.72.

8-Methyl-4, 5, 10,11 - te tra hydro- $2 \mathrm{H}$ benzo[f]imidazo[5,1-a]isoquinolin-1-one $(2 \boldsymbol{b})$. Yield 52\%, $\mathrm{mp} 220-222^{\circ} \mathrm{C}$ (decomp.). IR (KBr) $v_{\max } / \mathrm{cm}^{-1}: 1680(\mathrm{C}=\mathrm{C})$, $1716(\mathrm{C}=\mathrm{O}), 3270(\mathrm{~N}-\mathrm{H}) .{ }^{1} \mathrm{H}$ NMR $\left(\mathrm{CDCl}_{3}, 300 \mathrm{MHz}\right)$ : $\delta 2.33\left(\mathrm{~s}, 3 \mathrm{H}, \mathrm{CH}_{3}\right), 2.39$ (t, 2H, J 6.5, H-4), 2.84 (t, 2H, J 6.5, H-5), 3.41 (t, 2H, J 7.6, H-10), 3.87 (t, 2H, J 7.6, H-11), 6.30 (s, 1H, C=C-H), 7.50-7.72 (m, 3H, Ar-H), 8.54 (s, 1H, N-H). MS (EI, $m / z$ (rel.\%)): $252\left(\mathrm{M}^{+}, 80 \%\right), 251$ (20), 250 (35), 223 (20), 184 (17), 168 (40), 155 (16), 141 (42), 128 (33), 115

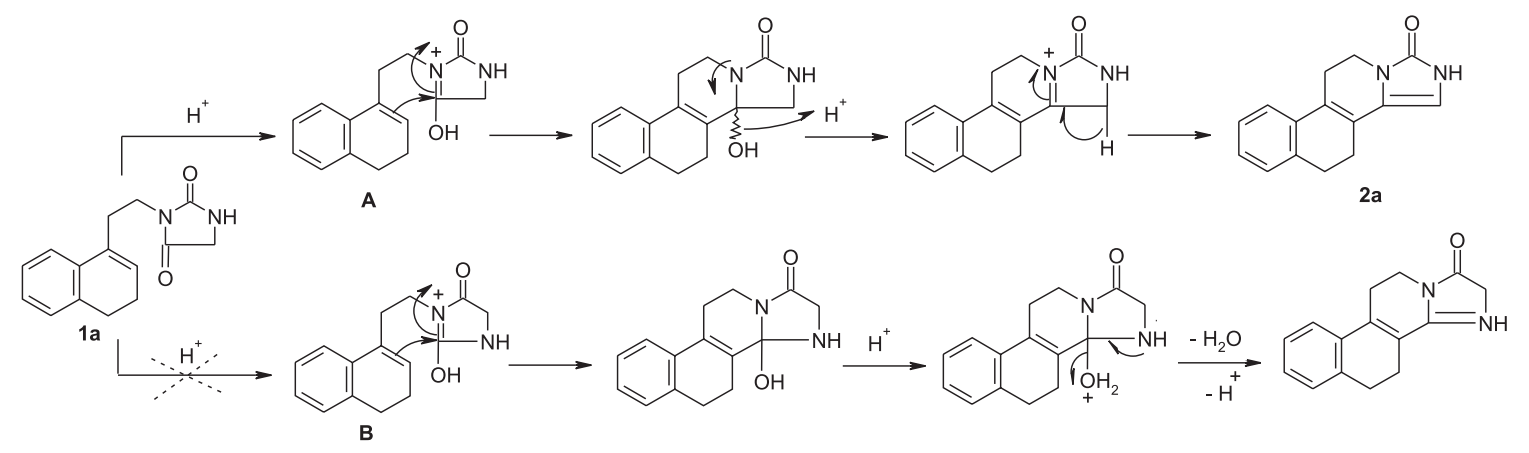

Scheme 2. Proposed mechanism for intramolecular cyclization of imidazolidine-2,4-diones. 
(58), 77 (100). UV $\left(\mathrm{CHCl}_{3}\right) \lambda_{\max } / \mathrm{nm}(\log \mathrm{e}): 288$ (3.79), 307 (3.85), 322 (3.98). Analysis calc. for $\mathrm{C}_{16} \mathrm{H}_{16} \mathrm{~N}_{2} \mathrm{O}: \mathrm{C}, 76.16$; H, 6.39; N, 11.10; Found: C, 76.19; H, 6.34; N, 11.14.

6-Chloro-4, 5, 10, 11 -tetrahydro- $2 \mathrm{H}$ benzolf]imidazo[5,1-a]isoquinolin-1-one (2c). Yield 42\%, $\mathrm{mp} 202-205^{\circ} \mathrm{C}$ (decomp.). IR (KBr) $v_{\max } / \mathrm{cm}^{-1}: 1670(\mathrm{C}=\mathrm{C})$, $1713(\mathrm{C}=\mathrm{O}), 3304(\mathrm{~N}-\mathrm{H}) .{ }^{1} \mathrm{H}$ NMR $\left(\mathrm{CDCl}_{3}, 200 \mathrm{MHz}\right): \delta$ 2.40 (t, 2H, J 6.6, H-4), 2.83 (t, 2H, J 6.6, H-5), 3.41 (t, 2H, $J 7.8, \mathrm{H}-10), 4.02(\mathrm{t}, 2 \mathrm{H}, J 7.8, \mathrm{H}-11), 6.62(\mathrm{~s}, 1 \mathrm{H}, \mathrm{C}=\mathrm{C}-\mathrm{H})$, 7.50-7.80 (m, 3H, Ar-H), 8.60 (s, 1H, N-H). UV $\left(\mathrm{CHCl}_{3}\right)$ $\lambda_{\max } / \mathrm{nm}(\log \varepsilon): 287$ (3.91), 304 (3.86), 321 (3.82). Analysis calc. for $\mathrm{C}_{15} \mathrm{H}_{13} \mathrm{~N}_{2} \mathrm{OCl}: \mathrm{C}, 66.06 ; \mathrm{H}, 4.80 ; \mathrm{N}, 10.27 ; \mathrm{Cl}$, 13.00; Found: C, 65.97; H, 4.82; N, 10.32; Cl, 12.94.

7,8-Dime thyl-4,5,10,11-tetrahydro-2 Hbenzolf]imidazo[5,1-a]isoquinolin-1-one (2d). Yield 59\%, $\mathrm{mp} 238-241^{\circ} \mathrm{C}$ (decomp.). IR (KBr) $v_{\max } / \mathrm{cm}^{-1}: 1670(\mathrm{C}=\mathrm{C})$, $1715(\mathrm{C}=\mathrm{O}), 3315(\mathrm{~N}-\mathrm{H}) .{ }^{1} \mathrm{H}$ NMR $\left(\mathrm{CDCl}_{3}, 60 \mathrm{MHz}\right)$ : $\delta$ 2.25-4.00 (m, 14H, aliphatic H), $6.43(\mathrm{~s}, 1 \mathrm{H}, \mathrm{C}=\mathrm{C}-\mathrm{H})$, 7.35 (s, 1H, Ar-H), 7.60 (s, 1H, Ar-H), 8.72 (s, 1H, NH). UV $\left(\mathrm{CHCl}_{3}\right) \lambda_{\text {max }} / \mathrm{nm}(\log \varepsilon): 292$ (3.93), 305 (3.87). Analysis calc. for $\mathrm{C}_{17} \mathrm{H}_{18} \mathrm{~N}_{2} \mathrm{O}: \mathrm{C}, 76.66 ; \mathrm{H}, 6.81 ; \mathrm{N}, 10.52$; Found: C, $76.75 ; \mathrm{H}, 6.78 ; \mathrm{N}, 10.50$.

10, 11 -Dihydro-2H-benzo[f]imidazo[5,1-a]isoquinolin-1-one (2e). Yield 47\%, $\mathrm{mp} 252-255^{\circ} \mathrm{C}$ (decomp.). IR (KBr) $v_{\max } / \mathrm{cm}^{-1}: 1680(\mathrm{C}=\mathrm{C}), 1715(\mathrm{C}=\mathrm{O})$, $3270(\mathrm{~N}-\mathrm{H}) .{ }^{1} \mathrm{H}$ NMR $\left(\mathrm{CDCl}_{3}, 300 \mathrm{MHz}\right): \delta 3.44$ (t, $2 \mathrm{H}, J$ 6.4, H-10), 4.01 (t, 2H, J 6.4, H-11), 6.70 (s, 1H, C=C-H), 7.46- 7.83 (m, 5H, Ar-H), 8.00 (d, 1H, J 8.41, H-9), 8.91 (br s, $1 \mathrm{H}, \mathrm{N}-\mathrm{H})$. UV $\left(\mathrm{CHCl}_{3}\right) \lambda_{\text {max }} / \mathrm{nm}(\log \varepsilon): 288$ (3.83), 307 (3.81), 321 (3.75). Analysis calc. for $\mathrm{C}_{15} \mathrm{H}_{12} \mathrm{~N}_{2} \mathrm{O}: \mathrm{C}, 76.25$; H, 5.12; N, 11.86; Found: C, 76.15; H, 5.16; N, 11.80.

8-Methyl-10, 11 -dihydro-2H-benzo[f]imidazo[5,1-a]isoquinolin-1-one (2f). Yield 55\%, mp $229-231{ }^{\circ} \mathrm{C}$ (decomp.). IR (KBr) $v_{\max } / \mathrm{cm}^{-1}: 1680(\mathrm{C}=\mathrm{C}), 1715(\mathrm{C}=\mathrm{O})$, $3250(\mathrm{~N}-\mathrm{H}) .{ }^{1} \mathrm{H}$ NMR $\left(\mathrm{CDCl}_{3}, 300 \mathrm{MHz}\right): \delta 2.51$ (s, $3 \mathrm{H}$, $\mathrm{CH}_{3}$ ), 3.41 (t, 2H, J 6.3, H-10), 4.00 (t, 2H, J 6.3, H-11), 6.66 (s, 1H, C=C-H), 7.38-7.98 (m, 5H, Ar-H), 8.90 (br s, $1 \mathrm{H}, \mathrm{N}-\mathrm{H})$. UV $\left(\mathrm{CHCl}_{3}\right) \lambda_{\max } / \mathrm{nm}(\log \varepsilon): 288$ (3.89), 307 (3.84), 322 (3.79). Analysis calc. for $\mathrm{C}_{16} \mathrm{H}_{14} \mathrm{~N}_{2} \mathrm{O}$ : C, 76.78; H, 5.64; N, 11.19; Found: C, 76.88; H, 5.66; N, 11.24.

6-Chloro-10,11-dihydro-2H-benzo[f]imidazo[5,1-a]isoquinolin-1-one (2g). Yield 45\%, mp 208-211 ${ }^{\circ} \mathrm{C}$ (decomp.). IR (KBr) $v_{\text {max }} / \mathrm{cm}^{-1}: 1680(\mathrm{C}=\mathrm{C}), 1710(\mathrm{C}=\mathrm{O}), 3200$ (N-H). ${ }^{~} \mathrm{HNMR}\left(\mathrm{CDCl}_{3}, 300 \mathrm{MHz}\right): \delta 3.43(\mathrm{t}, 2 \mathrm{H}, J 6.5, \mathrm{H}-10)$, 4.03 (t, 2H, J6.5, H-11), 6.68 (s, 1H, C=C-H), 7.60-7.92 (m, 4H, Ar-H), 8.00 (d, 1H, J8.41, H-9), 8.97 (br s, 1H, N-H). UV $\left(\mathrm{CHCl}_{3}\right)$ $\lambda_{\max } / \mathrm{nm}(\log \varepsilon)$ : 288 (3.92), 305 (3.90), 321 (3.85). Analysis calc. for $\mathrm{C}_{15} \mathrm{H}_{11} \mathrm{~N}_{2} \mathrm{OCl}$ : C, 66.55; H, 4.10; N, 10.35; Cl, 13.10; Found: C, 66.48; H, 4.14; N, 10.31; Cl, 13.07.

7,8-Dimethyl-10,11-dihydro-2H-benzo[f]imidazo[5,1-a]isoquinolin-1-one $(\mathbf{2 h})$. Yield $60 \%, \mathrm{mp} 245-247^{\circ} \mathrm{C}$ (decomp.). IR (KBr) $v_{\max } / \mathrm{cm}^{-1}: 1680(\mathrm{C}=\mathrm{C}), 1715(\mathrm{C}=\mathrm{O})$,
$3260(\mathrm{~N}-\mathrm{H}) .{ }^{1} \mathrm{H} \mathrm{NMR}\left(\mathrm{CDCl}_{3}, 60 \mathrm{MHz}\right): \delta 2.51$ (s, $6 \mathrm{H}, 2 \times$ $\mathrm{CH}_{3}$ ), 3.33-4.08 (m, 4H, H-10 and H-11), 6.70-7.64 (m, 5H, $4 \mathrm{Ar}-\mathrm{H}$ and $\mathrm{C}=\mathrm{C}-\mathrm{H}), 8.82(\mathrm{br} \mathrm{s}, 1 \mathrm{H}, \mathrm{NH})$. UV $\left(\mathrm{CHCl}_{3}\right) \lambda_{\max }{ }^{\prime}$ nm (log $\varepsilon$ ): 288 (3.98), 307 (3.92), 322 (3.84). Analysis calc. for $\mathrm{C}_{17} \mathrm{H}_{16} \mathrm{~N}_{2} \mathrm{O}: \mathrm{C}, 77.25 ; \mathrm{H}, 6.10 ; \mathrm{N}, 10.60$; Found: $\mathrm{C}$, 77.15; H, 6.14; N, 10.62 .

\section{Acknowledgements}

We are very much thankful to reviewers for their very helpful suggestions. JAP is thankful to UGC, New Delhi for the financial assistance.

\section{References}

1. Taylor, E. C.; Lenard, K.; J. Chem. Soc., Chem. Commun. 1967, 97.

2. Shibata, K.; Takeguwa, S.; Koizumi, N.; Yamakoshi, N.; Shimazawa, E.; Chem. Pharm. Bull. 1992, 40, 935.

3. Akherm, A. A.; Lakhvich, F. A.; Pshenichnyi, V. N.; Lis, L. G.; Kuzmitskii, B. B.; Mizulo, N. A.; USSR 636236 1978. (CA 90:104210t)

4. Norman, F. P.; Doorenbos, N. J.; J. Miss. Acad. Sci. 1976, 21, 23. (CA 86:165794a)

5. Anastasiou, A.; Catsoulacus, P.; Epitheor, K.; Farmakol. Farmakokinet. Intl. Ed. 1992, 6, 130. (CA 119:72911w)

6. Patrick, G. L.; Kinsman, O. S.; Eur. J. Med. Chem. 1996, 31, 615.

7. William, R. H.; Hoehn, M. M.; Michel, K. H.; US 147808 1994. (CA 123:75620u)

8. Greenbalatt, R. B.; Bornstan, R.; Bohler, C. S. S.; J. Reprod. Med. 1974, 13, 201.

9. Kierstead, R. W.; Faraone, A.; Boris, A.; J. Med. Chem. 1969, $12,629$.

10. Akherm, A. A.; Lakhvich, F. A.; Pshenichnyi, V. N.; Lakhvich, O. F.; Kuzmitskii, B. B.; Gorbatenko, S. F.; USSR 636235 1978. (CA 90:104211u)

11. Li, X.; Singh, S. M.; Lourdusamy, M.; Merand, Y.; Veitleux, R.; Labrie, F.; Bioorg. Med. Chem. Lett. 1995, 5, 1061.

12. Bakshi, R. K.; Patel, G. F.; Rasmussan, G. H.; US 373341, 1995. (CA 123:212693c)

13. Parihar, J. A.; Ramana, M. M. V.; Tetrahedron Lett. 2003, 44, 1843.

14. Parihar, J. A.; Ramana, M. M. V.; Chin. J. Chem. 2004, 22, 1196.

15. Kallies, B.; Mitzner, R.; J. Mol. Struct. (THEOCHEM). 1998, $428,267$.

16. Kallies, B; Mitzner, R.; J. Mol. Modeling. 1998, 4, 183.

17. Schleigh, W. R.; Catala, A.; Popp, F. D.; J. Heterocycl. Chem. 1965, 2, 379 .

Received: October 29, 2004 Published on the web: June 09, 2005 